\title{
Some Results on Relay Strategies for Memoryless Two-Way Relay Channels
}

\author{
Tao Cui and Tracey Ho \\ Department of Electrical Engineering \\ California Institute of Technology \\ Pasadena, CA 91125, USA \\ Email: $\{$ taocui, tho\}@caltech.edu
}

\author{
Jörg Kliewer \\ Klipsch School of Electrical and Computer Engineering \\ New Mexico State University \\ Las Cruces, NM 88003, USA \\ Email: jkliewer@nmsu.edu
}

\begin{abstract}
We consider relaying strategies for memoryless two-way relay channels, where the signal transmitted by a relay depends only on its previously received signal. For binary antipodal signaling under the general case of different SNRs on the terminal-relay channels, we consider two classes of relay strategies: abs-based strategies in which the processing at the relay is solely based on the absolute value of the received signal, and non-abs-based strategies. We analyze and optimize the performance of existing and new schemes for two-way relay channels under an average power constraint, including abs-based and non-abs-based versions of amplify and forward (AF), decode and forward (DF), and estimate and forward (EF). Additionally, we optimize the relay function via functional analysis such that the average probability of error is minimized in the high signalto-noise ratio (SNR) regime. Finally, we show that all these results can also be generalized to higher order constellations.
\end{abstract}

\section{INTRODUCTION}

Recently, the two-way communication problem in which both parties simultaneously transmit information to each other has gained a lot of attention in the literature. The two-way channel was first considered by Shannon [1], who derived inner and outer bounds on the capacity region. Later, the twoway relay channel (TWRC) has drawn renewed interest from both academic and industrial communities [2-6] due to its potential application in cellular and peer-to-peer networks. AF and DF protocols for one-way relay channels are extended to the half-duplex Gaussian TWRC in [2] and the general fullduplex discrete TWRC in [3]. In [4], network coding [7], [8] is used to increase the sum-rate of two users. With network coding, each node in a network is allowed to perform algebraic operations on received packets instead of only forwarding or replicating received packets. Works [2-4] focus on capacity bounds and their strategies are similar to those for one-way relay channels [9].

In this paper, we consider TWRCs with memoryless relay operation and average node power constraints. We first consider binary antipodal signaling by the terminals. A class of so called abs-based relaying schemes, where the processing at the relay is based on the absolute value of the received signal, is proposed in [10] for the special case of identical

This work has been supported in part by DARPA grant N66001-06-C2020, Caltech's Lee Center for Advanced Networking, the Okawa Foundation Research Grant and a gift from Microsoft Research.
SNRs on the source-relay channel. In the following we remove this limitation and consider the general case of different SNR for the terminal-relay channels. We analyze and optimize the symbol error probability of abs and non-abs relay strategies, not considering the effect of any end-to-end channel coding that may be applied. These include existing non-abs amplify and forward (AF) and decode and forward (DF) schemes for TWRCs, and their abs-based analogs, abs AF (AAF) and abs DF (ADF). Furthermore, we extend the estimate and forward (EF) strategy in [11] for the one-way relay channel to TWRCs. Besides characterizing the performance of different schemes, we also optimize the relay function via functional analysis, where the solution minimizes the average probability of error over all possible relay functions in the high-SNR regime. We show that the non-abs DF performs better than ADF if the two-way channel is very asymmetric or the relay has a greater power than the two terminals, while ADF performs better than DF in less asymmetric channels or when the relay has roughly the same power as the terminals. These results are also generalized to higher order constellations such as quadrature amplitude modulation (QAM) and pulse amplitude modulation (PAM).

Notations: In the following $\mathcal{N}\left(x, \sigma^{2}\right)$ denotes the Gaussian distribution $\frac{1}{\sqrt{2 \pi \sigma^{2}}} \exp \left(-\frac{x^{2}}{2 \sigma^{2}}\right)$ and $Q(\cdot)$ represents the Qfunction.

\section{SySTEM MODEL}

An example for a two-way communication over a relay channel with two terminals and a relay is displayed in Fig. 1, where the $X_{i}$ are the transmitted symbols from some given constellation at terminal $i, i=1,2, Y_{i}$ the received symbols at the terminals, and $Y_{R}$ is the transmitted symbol at the relay. The communication from terminal 1 to terminal 2 takes place in two transmission phases. In the multiple-access (MAC) phase, both terminals simultaneously send a block of data symbols to the relay which generates $Y_{R}=f\left(h_{1} X_{1}+h_{2} X_{2}+N\right)$ with the decoding function $f(\cdot)$. Here, $h_{1}$ and $h_{2}$ represent deterministic attenuation factors for the terminal-to-relay and relay-to-terminal channels, which could for example represent a single realization of a fading process. Throughout this paper, we assume that $h_{1} \geq h_{2}$ without loss of generality. The quantity $N$ represents the additive white Gaussian noise 


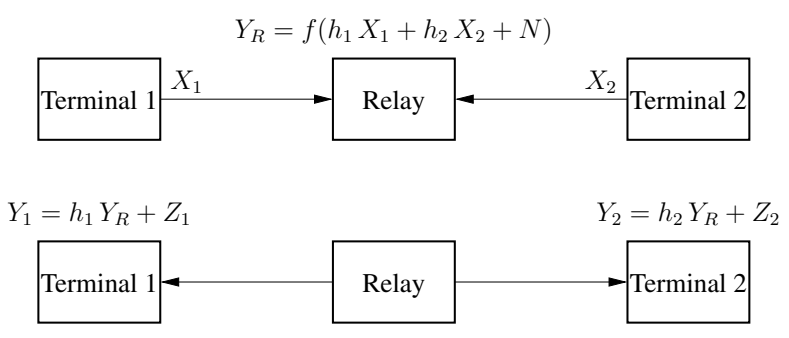

Fig. 1. Two-way relay channel.

(AWGN) at the relay with mean zero and variance $\sigma_{r}^{2}$. In the broadcast phase, the relay transmits $Y_{R}$ to both terminals 1 and 2, where $Z_{i}$ is the AWGN at terminal $i$ with mean zero and variance $\sigma_{s_{i}}^{2}$. The discrete-time model for the TWRC can therefore be written as

$$
Y_{i}=h_{i} f\left(h_{1} X_{1}+h_{2} X_{2}+N\right)+Z_{i}, \quad i=1,2 .
$$

For the sake of brevity we also define the received signal at the relay as $U=h_{1} X_{1}+h_{2} X_{2}+N$. Since each terminal knows what it has sent to the relay in the MAC phase, it can recover the information from the other terminal based on the received $Y_{i}$ and its own a priori symbols $X_{i}$. In addition, we impose an average power constraint on $X_{i}$ :

$$
E\left\{\left|X_{i}\right|^{2}\right\} \leq P_{s}, \quad i=1,2,
$$

as well as on the output of the relay:

$$
E\left\{\left|f\left(X_{1}+X_{2}+N\right)\right|^{2}\right\} \leq P_{r} .
$$

We assume for notational simplicity that the noise variance at the two terminals is the same, i.e., $\sigma_{s_{1}}^{2}=\sigma_{s_{2}}^{2}=\sigma_{s}^{2}$; extensions to the more general case are straightforward. We also assume that the terminals know $h_{1}$ and $h_{2}$, which may be obtained by using channel estimation at the relay or the feedback channel from the two terminals, see e.g., [12]. We focus on symbol error probability as a performance metric: each terminal is assumed to perform a hypothesis test to decide which symbol was transmitted by the other terminal; we do not consider the effect of any end to end channel coding that may be applied.

\section{RELAy STRATEGIES FOR BPSK IN ASYMMETRIC TWRC}

We begin by considering BPSK signaling at the terminals; an extension to higher order constellations is given in Section V. Each terminal transmits $X_{i}= \pm \sqrt{P_{s}}$. We consider two classes of relay strategies: absolute value strategies, where the relay transmits a function of $|U|$ [10], and non-absolute value strategies, where the relay transmits an odd function of $U$.

\section{A. Non-abs Based Strategies}

Here, we assume threshold decoding at the terminals where the following simple decision rule is applied: If $x_{i}=\sqrt{P_{s}}$ has been sent in the MAC phase then terminal $i$ decides on $\sqrt{P_{s}}$ if $y_{i} \geq v_{i}$ and on $-\sqrt{P_{s}}$ otherwise. Likewise, if $x_{i}=-\sqrt{P_{s}}$ has been sent, then terminal $i$ decides on $\sqrt{P_{s}}$ if $y_{i} \geq-v_{i}$ and on $-\sqrt{P_{s}}$ otherwise.
1) Amplify and Forward: With AF, the relay retransmits its received signal $u$, scaled to meet its power constraint.

2) Decode and Forward: In DF the relay performs hard decisions and maps each decision region to a fixed value that it transmits, i.e.,

$$
f(u)=\left\{\begin{array}{cc}
a, & \text { if } u \geq w, \\
b, & \text { if } w>u \geq 0 \\
-f(-u), & \text { otherwise }
\end{array}\right.
$$

where $w$ is a threshold to be determined. The average probability of error at terminal 1 can be written as

$$
\begin{aligned}
& P_{e}^{(1)}= \\
& \quad \frac{1}{2}+\frac{1}{2} \int_{0}^{w} A(u) d u \underbrace{\left[\int_{-\infty}^{v_{1}} \mathcal{N}\left(y-h_{1} b, \sigma_{s}^{2}\right) d y\right]}_{C\left(v_{1}, b\right)} \\
& +\frac{1}{2} \int_{w}^{+\infty} A(u) d u \underbrace{\left[\int_{-\infty}^{v_{1}} \mathcal{N}\left(y-h_{1} a, \sigma_{s}^{2}\right) d y\right]}_{D\left(v_{1}, a\right)} \\
& +\frac{1}{2} \int_{0}^{w} B(u) d u \underbrace{\left[\int_{-\infty}^{v_{1}} \mathcal{N}\left(y+h_{1} b, \sigma_{s}^{2}\right) d y\right]}_{E\left(v_{1}, b\right)} \\
& +\frac{1}{2} \int_{w}^{+\infty} B(u) d u \underbrace{\left[\int_{-\infty}^{v_{1}} \mathcal{N}\left(y+h_{1} a, \sigma_{s}^{2}\right) d y\right]}_{F\left(v_{1}, a\right)} .
\end{aligned}
$$

where

$$
\begin{gathered}
A(u)=\mathcal{N}\left(u-\left(h_{1}+h_{2}\right) \sqrt{P_{s}}, \sigma_{r}^{2}\right)- \\
\mathcal{N}\left(u-\left(h_{1}-h_{2}\right) \sqrt{P_{s}}, \sigma_{r}^{2}\right), \\
B(u)=\mathcal{N}\left(u+\left(h_{1}+h_{2}\right) \sqrt{P_{s}}, \sigma_{r}^{2}\right)- \\
\mathcal{N}\left(u+\left(h_{1}-h_{2}\right) \sqrt{P_{s}}, \sigma_{r}^{2}\right) .
\end{gathered}
$$

Likewise, the average probability of error at terminal 2 can be expressed by swapping the indices " 1 " and " 2 " in both (3) and (4). Taking the partial derivative of $P_{e}^{(1)}+P_{e}^{(2)}$ with respect to $w$ and setting the resulting equation to zero, we obtain

$$
\begin{aligned}
& A(w)\left(C\left(v_{1}, b\right)-D\left(v_{1}, a\right)\right)+ \\
& \quad B(w)\left(E\left(v_{1}, b\right)-F\left(v_{1}, a\right)\right)+\frac{\partial P_{e}^{(2)}}{\partial w}=0 .
\end{aligned}
$$

As the optimal solution of $w$ in (5) depends on $a, b, v_{1}, v_{2}$ in a complicated way, it is hard to solve (5) directly. A suboptimal solution to (5) can be approximated to be the root of $A(w)$, i.e., $w=h_{1} \sqrt{P_{s}}$. In high SNR, it is easy to verify that the first term on the left hand side of (5) is close to zero with this $w$. By substituting $w=h_{1} \sqrt{P_{s}}$ into (3), taking the partial derivative of (3) with respect to $v_{1}$, and setting the resulting equation to zero we obtain

$$
v_{1}=\frac{h_{1}(a+b)}{2}, \quad v_{2}=\frac{h_{2}(a-b)}{2} .
$$


In high SNR we can obtain the optimal $a$ and $b$ subject to the power constraint at the relay by substituting (6) into (3) and minimizing (2). This leads to the equivalent problem

$$
\begin{aligned}
\min _{a, b} \int_{\frac{a-b}{2} h_{1}}^{+\infty} \mathcal{N}(y) d y+\int_{\frac{\frac{a+b}{2} h_{2}}{+\infty} \mathcal{N}(y) d y,} \text { subject to } a^{2}+b^{2}=2 P_{r},
\end{aligned}
$$

which gives

$$
\frac{a}{b}=\frac{h_{1}+h_{2}}{h_{1}-h_{2}}, \quad a^{2}+b^{2}=2 P_{r} .
$$

Note that (8) agrees with the conventional DF, where the relay first find a point from the set $\left\{-h_{1}-h_{2},-h_{1}+h_{2}, h_{1}-h_{2}, h_{1}+h_{2}\right\}$ with the minimum Euclidean distance from the received signal and then transmits a scaled version of this point.

When $h_{1}=h_{2}$, (8) gives

$$
a=\sqrt{2 P_{r}}, \quad b=0 .
$$

In this strategy, the received signal at the relay is mapped to only three points instead of four points as mapping both $-h_{1}+h_{2}$ and $h_{1}-h_{2}$ to zero does not cause ambiguity at the two terminals. Thus, removing a constellation point results in power savings and performance improvements.

So far, we have only given suboptimal parameters setting. One way to approximate the optimal solution is to use an iterative method. At the beginning of the $k$-th iteration, assuming that $w^{(k)}$ is given $\left(w^{(0)}=h_{1} \sqrt{P_{s}}\right)$, we can choose $a^{(k)}, b^{(k)}, v_{1}^{(k)}, v_{2}^{(k)}$ by minimizing the average error probability. Then, $w^{(k+1)}$ can be obtained using $a^{(k)}, b^{(k)}, v_{1}^{(k)}, v_{2}^{(k)}$ from (5). The process repeats until convergence or the maximum number of iterations is achieved. From our experiments we find that less than five iterations are required before convergence.

From simulation results we find that when $h_{1} / h_{2}$ is close to one, (9) performs better than (8) and performs close to the optimal solution. As $h_{1} / h_{2}$ increases, (8) outperforms (9) in high SNR. But (9) still performs better than (8) in low SNR. One advantage of the suboptimal solutions (8) and (9) is that they do not depend on channel parameters and noise variances and are easy to implement in practice.

3) Estimate and Forward: In this strategy the relay transmits a scaled version of the MMSE estimate of $h_{1} X_{1}+h_{2} X_{2}$, i.e., we consider a function $g(u)$ such that

$$
g(u)=\underset{g^{\prime}(u)}{\arg \min } E\left\{\left|h_{1} x_{1}+h_{2} x_{2}-g^{\prime}(u)\right|^{2} \mid u\right\} .
$$

The objective function in (10) can be written as

$$
\begin{array}{r}
E\left\{\left|h_{1} x_{1}+h_{2} x_{2}-g(u)\right|^{2} \mid u\right\}= \\
\sum_{x_{1}, x_{2} \in\left\{-\sqrt{P_{s}}, \sqrt{P_{s}}\right\}} \frac{\operatorname{Pr}\left(u \mid x_{1}+x_{2}\right) \operatorname{Pr}\left(x_{1}+x_{2}\right)}{\operatorname{Pr}(u)} . \\
\left|h_{1} x_{1}+h_{2} x_{2}-g(u)\right|^{2} .
\end{array}
$$

By minimizing (11) over $g(u)$ we obtain (12) shown at the top of next page. The relay function $f(u)$ can then be acquired as a scaled version of $g(u)$. We find that $g(u)$ in (12) is close to the conventional DF (8) in high SNR.

4) Optimized Relay Function: The optimal relay function minimizes the sum of average probabilities of both terminals subject to the average power constraint. To solve the functional optimization problem, we first fix $v_{1}$ and $v_{2}$ and derive the relay function as a function of $v_{1}$ and $v_{2}$ via the Lagrange dual. Then the relay function is substituted into the objective function and the resulting equation is minimized over $v_{1}$ and $v_{2}$ by performing a line search around $v_{1}$ and $v_{2}$ in the optimal DF strategy. Since we do not have a convex optimization problem, the obtained solution may be a local optimum.

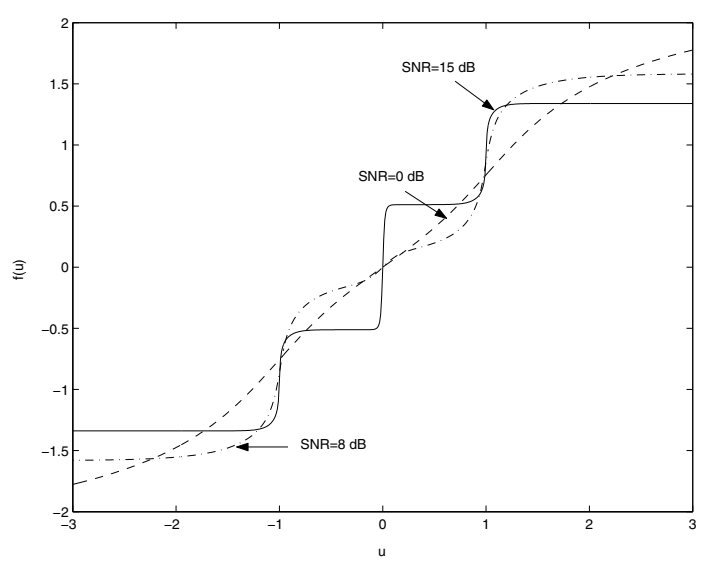

(a) $h_{1}=1$ and $h_{2}=0.5$

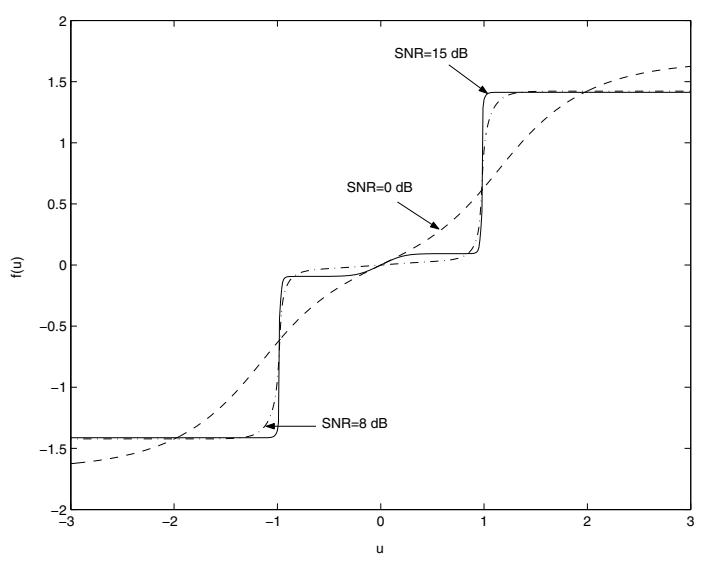

(b) $h_{1}=1$ and $h_{2}=0.8$

Fig. 2. The optimized non-abs relay function at different SNRs and with different $h_{1}$ and $h_{2}$.

Fig. 2 shows the optimized relay function at different SNRs and with different $h_{1}$ and $h_{2}$. In low SNR, the relay behaves like the AF strategy, while it looks like the DF strategy (8) in high SNR.

\section{B. Abs-Based Strategies}

Detailed derivations can be found in [10], where abs-based strategies are proposed and optimized for symmetric Gaussian 


$$
\begin{aligned}
g(u)=\frac{\sinh \frac{\left(h_{1}+h_{2}\right) \sqrt{P_{s}} u}{\sigma_{r}^{2}} e^{-\frac{\left(h_{1}+h_{2}\right)^{2} P_{s}}{2 \sigma_{r}^{2}}}\left(h_{1}+h_{2}\right) \sqrt{P_{s}}}{\cosh \frac{\left(h_{1}+h_{2}\right) \sqrt{P_{s}} u}{\sigma_{r}^{2}} e^{-\frac{\left(h_{1}+h_{2}\right)^{2} P_{s}}{2 \sigma_{r}^{2}}}+\cosh \frac{\left(h_{1}-h_{2}\right) \sqrt{P_{s}} u}{\sigma_{r}^{2}} e^{-\frac{\left(h_{1}-h_{2}\right)^{2} P_{s}}{2 \sigma_{r}^{2}}}} \\
+\frac{\sinh \frac{\left(h_{1}-h_{2}\right) \sqrt{P_{s}} u}{\sigma_{r}^{2}} e^{-\frac{\left(h_{1}-h_{2}\right)^{2} P_{s}}{2 \sigma_{r}^{2}}}\left(h_{1}-h_{2}\right) \sqrt{P_{s}}}{\cosh \frac{\left(h_{1}+h_{2}\right) \sqrt{P_{s}} u}{\sigma_{r}^{2}} e^{-\frac{\left(h_{1}+h_{2}\right)^{2} P_{s}}{2 \sigma_{r}^{2}}}+\cosh \frac{\left(h_{1}-h_{2}\right) \sqrt{P_{s}} u}{\sigma_{r}^{2}} e^{-\frac{\left(h_{1}-h_{2}\right)^{2} P_{s}}{2 \sigma_{r}^{2}}}} .
\end{aligned}
$$

two-way relay channels; the ADF strategy was previously proposed in [5]. In the following, we extend those strategies in [10] to asymmetric/fading channels.

1) Abs Amplify and Forward: In this scheme, the relay first takes the absolute value of the received signal and then subtracts a positive constant $C$ from the resulting signal, i.e.,

$$
f(u)=\beta(|u|-C),
$$

where $\beta$ is a coefficient to maintain the average power constraint at the relay, and $|u|$ denotes the absolute value of $u$. At terminal 1 , if the received signal is greater than the detection threshold $v$, it decides that $X_{1}=\sqrt{P_{s}}$ and $X_{2}=\sqrt{P_{s}}$ or $X_{1}=-\sqrt{P_{s}}$ and $X_{2}=-\sqrt{P_{s}}$, and it decides $X_{1}=\sqrt{P_{s}}$ and $X_{2}=-\sqrt{P_{s}}$ or $X_{1}=\sqrt{P_{s}}$ and $X_{2}=-\sqrt{P_{s}}$ otherwise. By minimizing the average probability of error, the optimal $v$ and $C$ can be obtained.

2) Abs Decode and Forward: In ADF, to satisfy the relay's average power constraint, $\sqrt{P_{r}}$ and $-\sqrt{P_{r}}$ are transmitted, i.e.,

$$
f(u)=\left\{\begin{array}{rc}
\sqrt{P_{r}}, & \text { if }|u| \geq h_{1} \sqrt{P_{s}}, \\
-\sqrt{P_{r}}, & \text { otherwise. }
\end{array}\right.
$$

Compared with (13), in ADF, the relay performs hard decisions on the absolute value of the received signal rather than transmitting a scaled version. At both terminals, the signal from the other terminal is determined by comparing the received signal with the threshold 0 . The average error probability at terminal 1 can be written as

$$
\begin{aligned}
P_{e}^{(1)} & =\frac{1}{2}+\frac{1}{2} \int_{0}^{h_{1} \sqrt{P_{s}}}\left(\mathcal{N}\left(u-\left(h_{1}+h_{2}\right) \sqrt{P_{s}}, \sigma_{r}^{2}\right)\right. \\
& +\mathcal{N}\left(u+\left(h_{1}+h_{2}\right) \sqrt{P_{s}}, \sigma_{r}^{2}\right)-\mathcal{N}\left(u-\left(h_{1}-h_{2}\right) \sqrt{P_{s}}, \sigma_{r}^{2}\right) \\
& \left.-\mathcal{N}\left(u+\left(h_{1}-h_{2}\right) \sqrt{P_{s}}, \sigma_{r}^{2}\right)\right) d u \\
& \cdot \int_{-\infty}^{0}\left(\mathcal{N}\left(y+h_{1} \sqrt{P_{r}}, \sigma_{s}^{2}\right)-\mathcal{N}\left(y-h_{1} \sqrt{P_{r}}, \sigma_{s}^{2}\right)\right) d y .
\end{aligned}
$$

The average error probability at terminal 2 can be obtained in the same way.

3) Estimate and Forward: In abs EF, we consider the function $g(u)$ such that

$$
g(u)=\underset{g^{\prime}(u)}{\arg \min } E\left\{|| h_{1} x_{1}+h_{2} x_{2}\left|-g^{\prime}(u)\right|^{2} \mid u\right\} .
$$

As in the non-abs case, by solving (16) we obtain (17), shown at the top of next page. The relay function $f(u)$ is then a scaled version of $g(u)-C$, where $C$ is a constant as in AAF.

4) Optimized Relay Function: The abs relay function can be optimized by following the approach as in the non-abs counterpart by minimizing the average probability of error over all possible functions $f(\cdot)$. The only difference is that in abs-based strategies we assume $f(\cdot)$ to be an even function.

The average probability of error at terminal 1 can be obtained as

$$
\begin{aligned}
& P_{e}^{(1)}(f)= \frac{1}{2} \int_{-\infty}^{+\infty} \mathcal{N}\left(u-\left(h_{1}-h_{2}\right) \sqrt{P_{s}}, \sigma_{r}^{2}\right) . \\
& {\left[\int_{v_{1}}^{+\infty} \mathcal{N}\left(y-h_{1} f(u), \sigma_{s}^{2}\right) d y\right] d u } \\
&+ \frac{1}{2} \int_{-\infty}^{+\infty} \mathcal{N}\left(u-\left(h_{1}+h_{2}\right) \sqrt{P_{s}}, \sigma_{r}^{2}\right) \\
& \cdot\left[\int_{-\infty}^{v_{1}} \mathcal{N}\left(y-h_{1} f(u), \sigma_{s}^{2}\right) d y\right] d u
\end{aligned}
$$

where $v_{1}$ is the decision threshold at terminal 1 . We can similarly obtain $P_{e}^{(2)}(f)$, the average probability of error at terminal 2. By minimizing $P_{e}^{(1)}(f)+P_{e}^{(2)}(f)$ subject to the average power constraint, we can approximate the optimal relay function as in (19) shown at the top of next page. Herein, $\lambda$ is a parameter to satisfy the average power constraint and $W(\cdot)$ denotes Lambert's $\mathrm{W}$ function $W(x)$, which is defined as the solution to $W(x) e^{W(x)}=x$. It can be shown, as in the symmetric TWRC case [10], that this converges to the ADF strategy and is the globally optimal abs-based strategy in high SNR.

\section{Analytical Comparison Between Two Classes OF STRATEGIES IN HIGH SNR}

For $h_{1}>h_{2}$, the average error probability of ADF can be approximated by applying Chernoff bound-type arguments to (15) (and the corresponding expression for terminal 2) according to

$$
P_{e}^{(1)}+P_{e}^{(2)} \approx \frac{1}{2}\left(e^{-\frac{h_{1}^{2} P_{r}}{2 \sigma_{s}^{2}}}+e^{-\frac{h_{2}^{2} P_{r}}{2 \sigma_{s}^{2}}}\right)+e^{-\frac{h_{2}^{2} P_{s}}{2 \sigma_{r}^{2}}} .
$$

Likewise, we can approximate the average error probability of non-abs DF by using Chernoff bounds on (3) and (4) (and the 


$$
\begin{aligned}
g(u)=\frac{\left|h_{1}+h_{2}\right| \sqrt{P_{s}} e^{-\frac{\left(h_{1}+h_{2}\right)^{2} P_{s}}{2 \sigma_{r}^{2}}} \cosh \frac{\left(h_{1}+h_{2}\right) \sqrt{P_{s}} u}{\sigma_{r}^{2}}}{e^{-\frac{\left(h_{1}+h_{2}\right)^{2} P_{s}}{2 \sigma_{r}^{2}}} \cosh \frac{\left(h_{1}+h_{2}\right) \sqrt{P_{s}} u}{\sigma_{r}^{2}}+e^{-\frac{\left(h_{1}-h_{2}\right)^{2} P_{s}}{2 \sigma_{r}^{2}}} \cosh \frac{\left(h_{1}-h_{2}\right) \sqrt{P_{s}} u}{\sigma_{r}^{2}}}+ \\
\frac{\left|h_{1}-h_{2}\right| \sqrt{P_{s}} e^{-\frac{\left(h_{1}-h_{2}\right)^{2} P_{s}}{2 \sigma_{r}^{2}}} \cosh \frac{\left(h_{1}-h_{2}\right) \sqrt{P_{s}} u}{\sigma_{r}^{2}}}{\operatorname{losh} \frac{\left(h_{1}-h_{2}\right) \sqrt{P_{s} u}}{\sigma_{r}^{2}}} .
\end{aligned}
$$

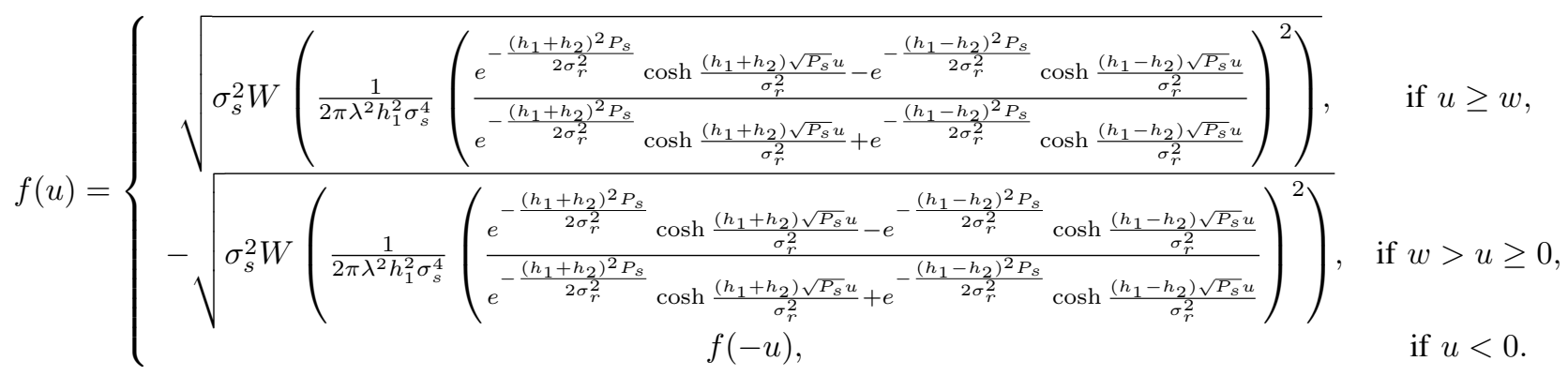

corresponding expression for terminal 2) as

$$
\begin{aligned}
& P_{e}^{(1)}+P_{e}^{(2)} \approx \\
& e^{-\frac{\beta^{2} h_{1}^{2} h_{2}^{2}}{2 \sigma_{s}^{2}}}+\frac{1}{2} e^{-\frac{h_{2}^{2} P_{s}}{2 \sigma_{r}^{2}}}\left(1+\frac{1}{2} Q\left(\frac{\beta h_{1} h_{2}-2 \beta h_{2}^{2}}{\sigma_{r}}\right)\right),
\end{aligned}
$$

where

$$
\beta=\sqrt{\frac{P_{r}}{h_{1}^{2}+h_{2}^{2}}} .
$$

In the following, we consider several cases in high SNR.

- If $\frac{P_{r}}{\sigma_{s}^{2}}<\frac{P_{s}}{\sigma_{r}^{2}},(20)$ is dominated by $\frac{1}{2} e^{-\frac{h_{2}^{2} P_{r}}{2 \sigma_{s}^{2}}}$ while (21) is dominated by $e^{-\frac{h_{1}^{2} h_{2}^{2} P_{r}}{2\left(h_{1}^{2}+h_{2}^{2}\right) \sigma_{r}^{2}}}$. Therefore, the average error probability of $\mathrm{ADF}$ is at least $1 / 2$ of that of $\mathrm{DF}$.

- If $h_{1}>2 h_{2}$ and $\frac{\beta^{2} h_{1}^{2}}{\sigma_{s}^{2}}>\frac{P_{s}}{\sigma_{r}^{2}},(20)$ is dominated by $e^{-\frac{h_{2}^{2} P_{s}}{2 \sigma_{r}^{2}}}$ and (21) is dominated by $\frac{1}{2} e^{-\frac{h_{2}^{2} P_{s}}{2 \sigma_{r}^{2}}}$. Therefore, the average error probability of DF is $1 / 2$ of that of ADF.

- If $h_{1}<2 h_{2}$ and $\frac{\beta^{2} h_{1}^{2}}{\sigma_{s}^{2}}>\frac{P_{s}}{\sigma_{r}^{2}}$, (20) is dominated by $e^{-\frac{h_{2}^{2} P_{s}}{2 \sigma_{r}^{2}}}$ and (21) is dominated by $\frac{3}{4} e^{-\frac{h_{2}^{2} P_{s}}{2 \sigma_{r}^{2}}}$. Therefore, the average error probability of DF is $3 / 4$ of that of ADF.

These results suggest that when the channel is very asymmetric or the relay has a greater power than the terminals we should use DF. When relay has almost the same power as the terminals we prefer ADF where the power savings by using the abs operation has a big impact on the overall performance. Note that from Section III-A2 we know that when $h_{1} / h_{2}$ is close to 1, DF with (9) performs better than DF with (8). Therefore, when the channel is symmetric and the relay has a greater power than the terminals we should use DF with (9).

\section{Higher ORder CONSTEllations}

In practical standards such as the 802.11 series, usually higher order QAM constellations are employed to achieve high spectral efficiency. In the following, we assume $h_{1}=h_{2}=1$ for simplicity. We first define a mapping function $h(u)$ at the relay such that in the noise free case, each terminal can decode the other terminal's signal given its transmitted signal. This is equivalent to

$$
\begin{array}{lll}
h\left(u_{1}+u_{2}\right) \neq h\left(u_{1}^{\prime}+u_{2}\right), & \forall u_{1} \neq u_{1}^{\prime} & \text { and } \\
h\left(u_{1}+u_{2}\right) \neq h\left(u_{1}+u_{2}^{\prime}\right), & \forall u_{2} \neq u_{2}^{\prime}, & u_{i}, u_{i}^{\prime} \in \mathcal{V},
\end{array}
$$

$i=1,2$, where $\mathcal{V}$ is the constellation set used by the two terminals. Mappings $h(u)$ satisfying the decodability condition (22) can be viewed as vertex colorings of an undirected graph $\mathcal{G}$, where each node corresponds to a different value of $u_{1}+u_{2}$ and there is an edge between the node corresponding to $u_{1}+u_{2}$ and the node corresponding to $u_{1}^{\prime}+u_{2}, \forall u_{1}^{\prime} \neq u_{1}$. Each such mapping $h(u)$ corresponds to a vertex coloring of $\mathcal{G}$ such that any pair of adjacent nodes does not have the same color. The relay constellation size is equal to the number of colors. The minimum relay constellation size, which equals the chromatic number of $\mathcal{G}$, is at least $|\mathcal{V}|$. For example, if the terminals use an 8-PSK constellation for the uplink channel to the relay, we obtain from a computer search that the minimum possible constellation size is eight for the downlink channel ${ }^{1}$.

The classification of BPSK strategies into absolute and nonabsolute value strategies can be generalized to a classification based on underlying relay mappings $h(u)$ satisfying (22). For each such mapping, the strategies discussed for BPSK in Section III-A and Section III-B can be generalized as described

\footnotetext{
${ }^{1}$ For arbitrary constellations $\mathcal{V}$ the minimum possible relay constellation size can be larger than $|\mathcal{V}|$.
} 
below. Under different channel parameters, different mappings achieve the lowest error probability.

Note that rectangular QAM constellations can be easily transmitted as two PAM signals on quadrature carriers. In the following, we take 4PAM as an example. The approach can be generalized to higher PAM constellations. For simplicity, we assume that the transmit signal by the relay is chosen from the constellation set $\mathcal{V}=\{-3,-1,1,3\}$. In the absence of noise, the received signal at the relay is from the set $\mathcal{W}=\{-6,-4,-2,0,2,4,6\}$. The underlying (noise free) relay mapping $h(u)$ maps the set $\mathcal{W}$ to a set $\mathcal{V}^{\prime}$ of size at least $|\mathcal{V}|$. We first consider the class of mapping functions such that they map $\mathcal{W}$ to $\mathcal{V}^{\prime}=\mathcal{V}$. For example, we can choose

$$
\begin{aligned}
& h(-6)=-3, \quad h(-4)=-1, \quad h(-2)=3, \quad h(0)=1, \\
& h(2)=-3, \quad h(4)=-1, \quad h(6)=3,
\end{aligned}
$$

or

$$
\begin{aligned}
& h(-6)=-3, \quad h(-4)=-1, \quad h(-2)=1, \quad h(0)=3, \\
& h(2)=-3, \quad h(4)=-1, \quad h(6)=1 .
\end{aligned}
$$

It is easy to verify that both (23) and (24) satisfy the condition in (22). Note that (24) is the physical network coding operation given in [5] using DF.

AAF can be readily generalized by setting the relay function to be a piecewise linear function based on $h(u)$ such as

$$
f(u)=\left\{\begin{array}{cl}
\beta(u+3), & \text { if } u<-3, \\
\beta(u+5), & \text { if }-2>u \geq-3, \\
\beta(1-u), & \text { if } 1>u \geq-2, \\
\beta(-1-u), & \text { if } 2>u \geq 1, \\
\beta(u-5), & \text { if } 5>u \geq 2, \\
\beta(u-3), & \text { if } u \geq 5,
\end{array}\right.
$$

where $\beta$ is a coefficient to maintain the average power constraint at the relay. The decoding at each terminal is similar to the traditional 4PAM demodulation by comparing with some thresholds. We can optimize $d_{i}$ and $c_{i}$ in the relay function and the thresholds at the terminals by minimizing the average error probability. ADF can be adapted similarly. The relay defines hard decision regions for $u$, and sends a scaled/shifted version of $h(u)$. In high SNR, the ADF relay function based on (23) can be obtained as

$$
f(u)=\left\{\begin{array}{cl}
-3 \beta, & \text { if } u<-5 \\
-\beta, & \text { if }-3>u \geq-5, \\
3 \beta, & \text { if }-1>u \geq-3, \\
\beta, & \text { if } 1>u \geq-1 \\
-3 \beta, & \text { if } 3>u \geq 1 \\
-\beta, & \text { if } 5>u \geq 3 \\
3 \beta, & \text { if } u \geq 5
\end{array}\right.
$$

For EF, we first consider the function $g(u)$ such that

$$
g(u)=\underset{g^{\prime}(u)}{\arg \min } E\left\{\left|h\left(x_{1}+x_{2}\right)-g^{\prime}(u)\right|^{2} \mid u\right\} .
$$

$f(u)$ is then a scaled version of $g(u)$, i.e., $f(u)=\beta g(u)$, where $\beta \geq 0$ is a scalar to satisfy the average power constraint.
At the two terminals, there also exists an optimal decision threshold $v$. We can optimize $v$ using the same approach as in AAF or just choose the conventional 4PAM detection threshold. In all strategies, we can also apply a maximum likelihood detector at each terminal, i.e.,

$$
\hat{x}_{2}=\underset{\tilde{x}_{2} \in \mathcal{V}}{\arg \min }\left|y_{1}-f\left(x_{1}+\tilde{x}_{2}\right)\right|^{2} .
$$

The relay mapping function can also perform a redundant mapping such that $\mathcal{W}=\{-6,-4,-2,0,2,4,6\}$ is mapped to a set $\mathcal{V}^{\prime}$ with 5,6 , or 7 elements. For example, when $\mathcal{V}^{\prime}=$ $\{-4,-2,0,2,4\}$, we can choose

$$
\begin{aligned}
& h(-6)=-4, \quad h(-4)=-2, \quad h(-2)=0, \quad h(0)=2, \\
& h(2)=4, \quad h(4)=-2, \quad h(6)=-4,
\end{aligned}
$$

or, when $\mathcal{V}^{\prime}=\{-5,-3,-1,1,3,5\}$, we can choose

$$
\begin{aligned}
& h(-6)=-5, \quad h(-4)=-3, \quad h(-2)=-1, \quad h(0)=1, \\
& h(2)=3, \quad h(4)=5, \quad h(6)=-5 .
\end{aligned}
$$

When $\mathcal{V}^{\prime}=\mathcal{W}$, we can simply choose $h(u)=u$. It is easy to verify that (29) and (30) satisfy the condition in (22).

\section{Vi. Simulation Results}

Fig. 3 compares the bit error rate (BER) performance of different abs and non-abs strategies for BPSK when $h_{1}=1$ and $h_{2}=0.8, P_{r}=P_{s}=1$. We observe that in low SNR, the optimized non-abs (abs) relay behaves like the AF (AAF) strategy, while it looks like the DF (ADF) strategy in high SNR. Also, EF performs close to the optimized strategy for all SNR values. It can also be seen that non-abs-based strategies perform better than abs-based strategies in low SNR in this scenario while the former performs worse than the latter in high SNR. The reason for this is that non-abs strategies do not exploit the fact that a priori information is available at the terminals and therefore provide extra redundancy which is useful particularly in low SNR. A similar behavior is observed in Fig. 4 where the case $h_{1}=1$ and $h_{2}=0.5$ is considered. Compared to the results for $h_{1}=1$ and $h_{2}=0.8$ in Fig. 3, the threshold SNR below which non-abs-based strategies perform better than abs-based strategies is increased. Thus, non-absbased strategies are beneficial for asymmetric channels.

Fig. 5 compares the symbol error rate (SER) of different relay functions for 4PAM using $\mathrm{ADF}$ and $\mathrm{AAF}$ when the relay constellation size $V=\left|\mathcal{V}^{\prime}\right|$ takes values $4,5,6,7$, for moderate to high SNR. We can see that the performance degrades as $V$ increases. There are two factors that may affect the performance of relay functions with different $V$. First, a small $V$ indicates a higher compression at the relay, which results in power savings. Second, when $V$ is small, a decoding error at the relay may affect the overall performance. In high SNR, it is clear that the power savings dominate the performance of ADF. We can also see that the mappings in (23) and (24), which have equal $V$, give practically identical performance. In low SNR, we find that the performance degrades as $V$ 


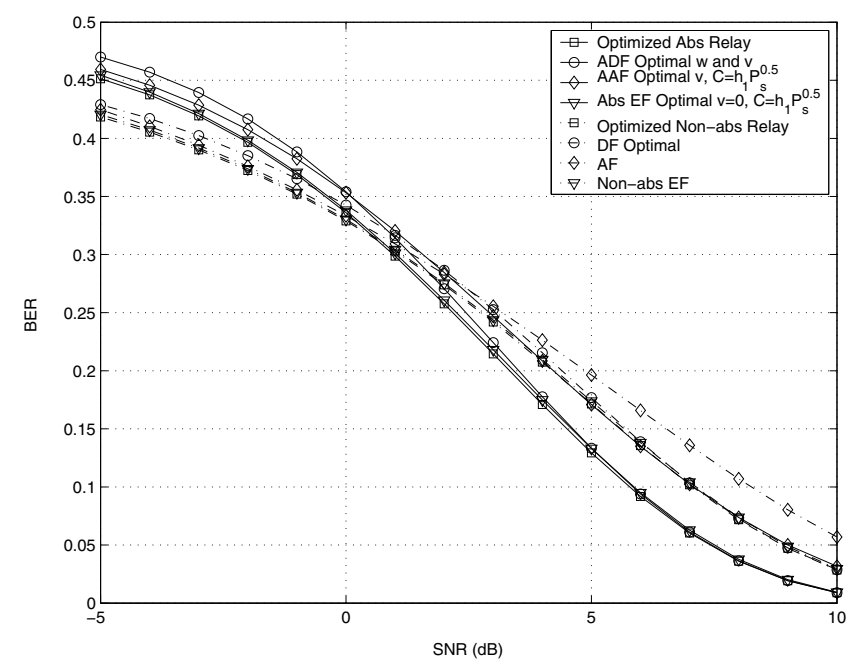

Fig. 3. Performance comparison between different abs and non-abs strategies when $h_{1}=1$ and $h_{2}=0.8, P_{r}=P_{s}=1$.

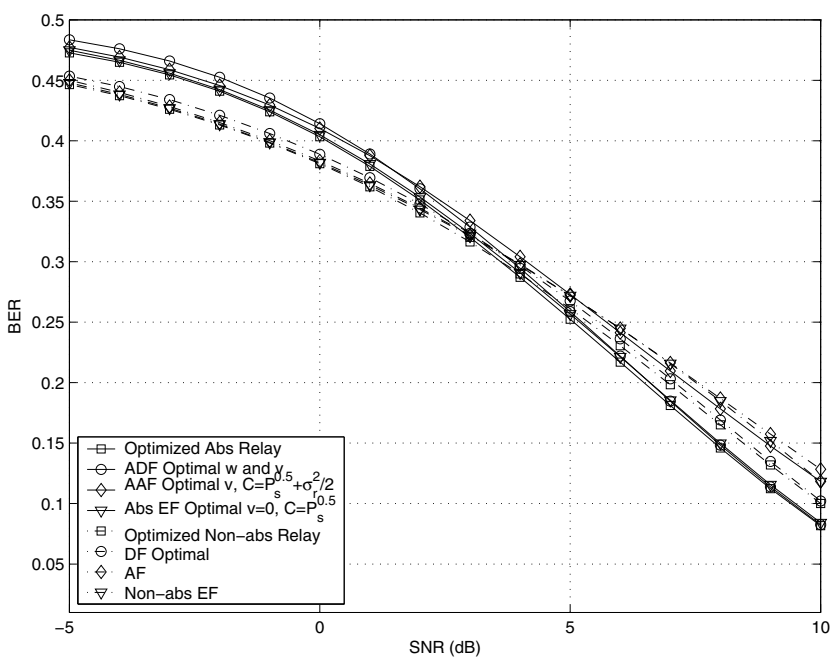

Fig. 4. Performance comparison between different abs and non-abs strategies when $h_{1}=1$ and $h_{2}=0.5, P_{r}=P_{s}=1$.

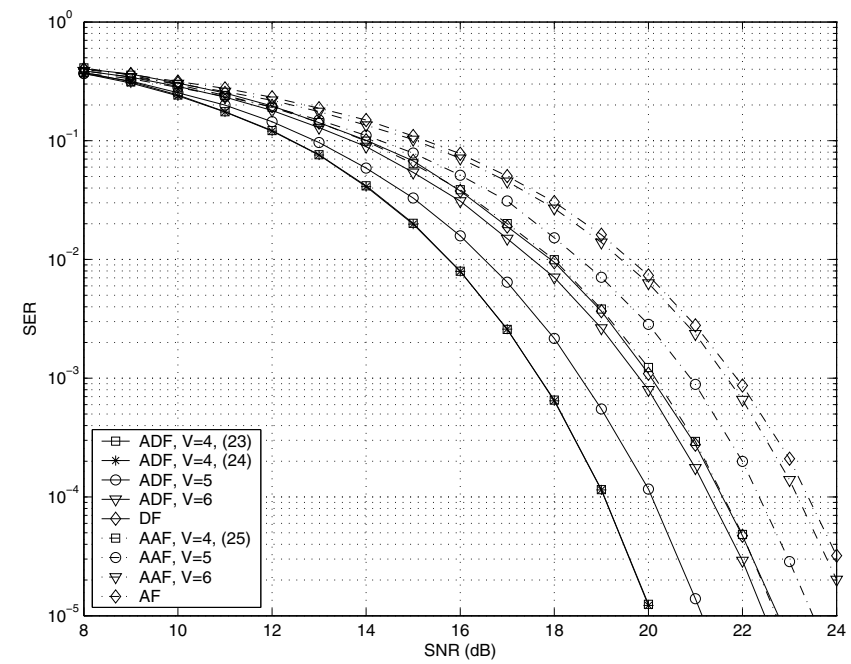

Fig. 5. SER comparison of ADF and AAF relay functions for 4PAM with $V=|\mathcal{V}|=4,5,6,7$ and $\sigma_{r}^{2}=\sigma_{s}^{2}$. decreases, which means that $V=7$ achieves the best performance. For example, at $\mathrm{SNR}=0 \mathrm{~dB}$, the SERs for $V=4$, $5,6,7$ are $0.6904,0.6472,0.6428,0.6146$, respectively. This observation generalizes that in the BPSK case, where the absbased functions correspond to the minimum relay constellation size. The reason for this behavior is again that the redundancy in the relay constellation set increases for larger $V$.

\section{CONCLUSION}

We have analyzed and optimized relaying strategies for memoryless TWRCs. In particular, we propose abs-based strategies where the relay processes the absolute value of the received signal. These techniques generally outperform non-abs-based strategies in the moderate to high SNR regime since they take into account that side information is available at the terminals which allows for additional power savings. Specifically, we have considered abs- and non-abs-based DF, $\mathrm{AF}$ and EF strategies, and also the optimization of the nonlinear processing function at the relay. In addition, we showed analytically for the high SNR case that the non-abs DF performs better than ADF when the two-way channel is very asymmetric or the relay has a greater power than the two terminals, while ADF performs better than DF when the relay has roughly the same power as the terminals. Although this work does not consider channel coding, the obtained expressions for the error probability allow rough determination of the required rate for an end-to-end channel code. Extensions of these results to higher order constellations such as QAM and PAM have also been presented, where similar observations can be made.

\section{REFERENCES}

[1] C. E. Shannon, "Two-way communication channels," in Proc. 4th Berkeley Symp. Math. Stat. Prob., 1961, pp. 611-644.

[2] B. Rankov and A. Wittneben, "Spectral efficient signaling for halfduplex relay channels," in Proc. of Asilomar Conference on Signals, Systems and Computers, Oct. 2005, pp. 1066-1071.

[3] — "Achievable rate regions for the two-way relay channel," in Proc. of IEEE ISIT, July 2006, pp. 1668-1672.

[4] C. Hausl and J. Hagenauer, "Iterative network and channel decoding for the two-way relay channel," in Proc. of IEEE ICC, June 2006, pp. $1568-1573$.

[5] S. Zhang, S. C. Liew, and P. P. Lam, "Hot topic: physical-layer network coding," in Proc. of ACM Mobicom, 2006, pp. 358-365.

[6] T. Cui and T. Ho, "Distributed space-time coding for two-way wireless relay networks," Caltech, Tech. Rep., Sept. 2007.

[7] R. Ahlswede, N. Cai, S. Y. R. Li, and R. W. Yeung, "Network information flow," IEEE Trans. Inform. Theory, vol. 46, no. 4, pp. 1204 1216, Jul. 2000 .

[8] T. Ho, M. Médard, R. Koetter, D. Karger, M. Effros, J. Shi, and B. Leong, "A random linear network coding approach to multicast," IEEE Trans. Inform. Theory, vol. 52, no. 10, pp. 4413-4430, Oct. 2006.

[9] J. Laneman, D. Tse, and G. Wornell, "Cooperative diversity in wireless networks: efficient protocols and outage behavior," IEEE Trans. Inform. Theory, vol. 50, no. 12, pp. 3062-3080, Dec. 2004.

[10] T. Cui, T. Ho, and J. Kliewer, "Relay strategies for memoryless twoway relay channels: Performance analysis and optimization," in Proc. of IEEE ICC, May 2008.

[11] K. S. Gomadam and S. A. Jafar, "Optimal relay functionality for SNR maximization in memoryless relay networks," IEEE J. Select. Areas Commun., vol. 25, no. 2, pp. 390-401, Feb. 2007.

[12] T. Cui, F. Gao, and A. Nallanathan, "Optimal training design for channel estimation in amplify and forward relay networks," in Proc. of IEEE Globecom, 2007. 